\title{
Balanced vertex decomposable simplicial complexes and their $h$-vectors
}

\author{
Jennifer Biermann \\ Department of Mathematics and Statistics \\ Mt. Holyoke College \\ South Hadley, MA, USA \\ jbierman@mtholyoke.edu
}

\author{
Adam Van Tuyl \\ Department of Mathematical Sciences \\ Lakehead University \\ Thunder Bay, ON, Canada \\ avantuyl@lakeheadu.ca
}

Submitted: July 14, 2012; Accepted: Jul 27, 2013; Published: Aug 9, 2013

Mathematics Subject Classifications: 05E45, 05A15, 13F55

\begin{abstract}
Given any finite simplicial complex $\Delta$, we show how to construct from a colouring $\chi$ of $\Delta$ a new simplicial complex $\Delta_{\chi}$ that is balanced and vertex decomposable. In addition, the $h$-vector of $\Delta_{\chi}$ is precisely the $f$-vector of $\Delta$. Our construction generalizes the "whiskering" construction of Villarreal, and Cook and Nagel. We also reverse this construction to prove a special case of a conjecture of Cook and Nagel, and Constantinescu and Varbaro on the $h$-vectors of flag complexes.
\end{abstract}

Keywords: simplicial complex, vertex decomposable, flag complex, $h$-vector

\section{Introduction}

The work of this paper was inspired by the "whiskering" construction of finite simple graphs found in work of Villarreal [21] and Cook and Nagel [7]. Given a finite graph $G=\left(V_{G}, E_{G}\right)$ on the vertex set $V_{G}=\left\{x_{1}, \ldots, x_{n}\right\}$, Villarreal constructed a new graph, denoted $G^{W}$, on the vertex set $\left\{x_{1}, \ldots, x_{n}, y_{1}, \ldots, y_{n}\right\}$ by adjoining the edges $\left\{x_{i}, y_{i}\right\}$ for every $i$ to the graph $G$. The new graph has a "whisker" at every vertex of the original graph. As discovered by Villarreal, the edge ideal of the new graph $G^{W}$, that is,

$$
I\left(G^{W}\right)=\left\langle w_{i} w_{j} \mid\left\{w_{i}, w_{j}\right\} \in E_{G^{W}}\right\rangle \subseteq R=k\left[x_{1}, \ldots, x_{n}, y_{1}, \ldots, y_{n}\right]
$$

has the property that $R / I\left(G^{W}\right)$ is Cohen-Macaulay. It was later observed by Dochtermann and Engström [8] and Woodroofe [22], and generalized by Cook and Nagel [7], that one could deduce this result by studying the topological properties of the simplicial complex associated to $I\left(G^{W}\right)$ via the Stanley-Reisner correspondence. In particular, Villarreal's 
construction can be viewed as creating a new independence complex $\Delta^{\prime}$ (sometimes called a flag complex) from the independence complex $\Delta$ of $G$. This new complex $\Delta^{\prime}$ is vertex decomposable (as defined by Provan and Billera [18]), and it is this topological property that implies that $R / I\left(G^{W}\right)$ is Cohen-Macaulay.

Our entry point is to ask whether there is a more general theory that can be applied to all simplicial complexes. Moreover, we want this general theory to specialize to known cases for flag complexes. In Section 3 we will show that a general construction exists using the notion of a colouring $\chi$ of a simplicial complex $\Delta$. From the colouring $\chi$ and complex $\Delta$, we make a new complex, denoted $\Delta_{\chi}$. Regardless of how one colours $\Delta$, the construction of $\Delta_{\chi}$ always results in a balanced vertex decomposable simplicial complex (see Theorem 7). It should be noted that this construction has appeared in several guises over the years (see Remark 4 and Discussion 11).

The consequences of our results are explored in Section 4. In particular, it is shown that the $f$-vector of a simplicial complex is also the $h$-vector of a balanced vertex decomposable simplicial complex. Although this result is implicit in work of Björner, Frankl, and Stanley [3], to the best of our knowledge, this fact has not explicitly appeared in the literature (although the case of flag complexes occurs in [7]). We also show that the graded Betti numbers of the Stanley-Reisner ideal of the Alexander dual of $\Delta_{\chi}$ can be expressed directly in terms of the $f$-vector of $\Delta$ (see Theorem 13).

Section 5 describes when our construction can be reversed, i.e., starting with a balanced vertex decomposable simplicial complex $\Delta$, we construct another simplicial complex $\Delta^{\prime}$ such that $f$-vector of $\Delta^{\prime}$ is the same as the $h$-vector of $\Delta$. We use this procedure to prove that the set of $f$-vectors of independence complexes of chordal graphs is precisely the set of $h$-vectors of balanaced vertex decomposable independence complexes of chordal graphs. Our result is a special case of a conjecture of Cook and Nagel [7] and Constantinescu and Varbaro [5] that the set of $f$-vectors of flag complexes is precisely the set of $h$-vectors of balanced vertex decomposable flag complexes.

As a final comment, we do not discuss the "whiskering" procedure found in [10] in which whiskers are added to only some of the vertices. This idea is explored in [2].

Acknowledgements. We thank Uwe Nagel and the referees for their invaluable comments. The authors made use of the computer programs CoCoA [4] and Macaulay 2 [13], including the Macaulay 2 package [6]. The second author acknowledges the support of NSERC.

\section{Background}

We work over the polynomial rings $S=k\left[x_{1}, \ldots, x_{n}\right]$ and $R=k\left[x_{1}, \ldots, x_{n}, y_{1}, \ldots, y_{s}\right]$ where $k$ is any field. Let $\Delta$ be a finite simplicial complex on vertex set $\left\{x_{1}, \ldots, x_{n}\right\}$ of dimension $d$. We say $\Delta$ is pure if all its facets (maximal elements) have the same cardinality. An important combinatorial invariant of $\Delta$ is its $f$-vector, that is, the vector $f(\Delta)=\left(f_{-1}, f_{0}, \ldots, f_{d}\right)$ where $f_{i}$ denotes the number of faces of $\Delta$ of dimension $i$. 
If $\sigma \in \Delta$, then the deletion of $\sigma$ is the simplicial complex $\Delta \backslash \sigma=\{\tau \in \Delta \mid \sigma \not \subset \tau\}$, and the link of $\sigma$ is $\operatorname{link}_{\Delta}(\sigma)=\{\tau \in \Delta \mid \sigma \cap \tau=\emptyset, \sigma \cup \tau \in \Delta\}$. When $\sigma=\{v\}$, we shall abuse notation and write $\Delta \backslash v$ (respectively $\operatorname{link}_{\Delta}(v)$ ). With this notation, we introduce a family of complexes due to Provan and Billera [18].

Definition 1. A pure simplicial complex $\Delta$ is called vertex decomposable if $(i) \Delta$ is a simplex, or $(i i)$ there exits $v \in V$ such that $\Delta \backslash v$ and $\operatorname{link}_{\Delta}(v)$ are vertex decomposable.

Key to the main construction studied in this paper is the notion of a colouring.

Definition 2. Let $\Delta$ be a simplicial complex on the vertex set $V$ with facets $F_{1}, \ldots, F_{t}$. An s-colouring of $\Delta$ is a partition of the vertices $V=V_{1} \cup \cdots \cup V_{s}$ (where the sets $V_{i}$ are allowed to be empty) such that $\left|F_{i} \cap V_{j}\right| \leqslant 1$ for all $1 \leqslant i \leqslant t, 1 \leqslant j \leqslant s$. We will sometimes write $\chi$ is an $s$-colouring of $\Delta$ to mean $\chi$ is a specific partition of $V$ that gives an $s$-colouring of $\Delta$. If there exists an $s$-colouring, we say that $\Delta$ is s-colourable. If $\Delta$ has dimension $d-1$, then we say that $\Delta$ is balanced if it is $d$-colourable.

We will be interested in how our results specialize to independence complexes of graphs, sometimes called flag complexes. Recall that if $G=\left(V_{G}, E_{G}\right)$ is a finite simple graph with vertex set $V_{G}=\left\{x_{1}, \ldots, x_{n}\right\}$ and edge set $E_{G}$, then a subset $W \subseteq V_{G}$ is an independent set of a graph $G$ if for every edge $e \in E_{G}$, we have $e \nsubseteq W$. The independence complex of $G$, denoted $\operatorname{Ind}(G)$, is the simplicial complex defined by

$$
\operatorname{Ind}(G)=\left\{W \subseteq V_{G} \mid W \text { is an independent set of } G\right\} .
$$

\section{A construction and its properties}

Starting with a simplicial complex $\Delta$ and an $s$-colouring $\chi$, we introduce a procedure to construct a new simplicial complex that is pure of dimension $s-1$, balanced, and vertex decomposable.

Construction 3. Let $\Delta$ be a simplicial complex on the vertex set $\left\{x_{1}, \ldots, x_{n}\right\}$. Given an $s$-colouring $\chi$ of $\Delta$ given by $V=V_{1} \cup \cdots \cup V_{s}$, we define $\Delta_{\chi}$ on vertex set

$\left\{x_{1}, \ldots, x_{n}, y_{1}, \ldots, y_{s}\right\}$ to be the simplicial complex with faces $\sigma \cup \tau$ where $\sigma$ is a face of $\Delta$ and $\tau$ is any subset of $\left\{y_{1}, \ldots, y_{s}\right\}$ such that for all $y_{j} \in \tau$ we have $\sigma \cap V_{j}=\emptyset$.

Remark 4. Construction 3 was introduced independently by Frohmader [11, Construction 7.1]. However, the construction appears implicitly in earlier work [3, Section 5], although it is only applied to compressed multicomplexes. Another variation appears in work of Hetyei (see [14, Definition 4.2]). The whiskering constructions found in [7, 21] for flag complexes become special cases of Construction 3. For example, Villarreal's construction in [21] of adding whiskers to every vertex of a graph $G$, and studying the resulting independence complex corresonds to the colouring $V=\left\{x_{1}\right\} \cup\left\{x_{2}\right\} \cup \cdots \cup\left\{x_{n}\right\}$ and applying Construction 3 to $\operatorname{Ind}(G)$. We point the reader to [11] which makes the connection to Cook and Nagel's clique whiskering more explicit. 
Observe that each $s$-colouring $\chi$ of $\Delta$ creates a new simplicial complex $\Delta_{\chi}$. Even though these simplicial complexes $\Delta_{\chi}$ may be different, they all share some interesting properties.

Theorem 5. The facets of $\Delta_{\chi}$ are in one-to-one correspondence with the faces of the original simplicial complex $\Delta$. In addition, $\Delta_{\chi}$ is pure of dimension $s-1$ and balanced.

Proof. Let $V=V_{1} \cup \cdots \cup V_{s}$ be the colouring of $\Delta$ given by $\chi$. From the definition of $\Delta_{\chi}$, the maximal faces are those of the form $\sigma \cup\left\{y_{j} \mid V_{j} \cap \sigma=\emptyset\right\}$ where $\sigma$ is a face of $\Delta$. This establishes the one-to-one correspondence.

If we partition the vertices of $\Delta_{\chi}$ as $\left\{x_{1}, \ldots, x_{n}, y_{1}, \ldots, y_{s}\right\}=V_{1}^{\prime} \cup V_{2}^{\prime} \cup \cdots \cup V_{s}^{\prime}$ where $V_{j}^{\prime}=V_{j} \cup\left\{y_{j}\right\}$, then this partition gives an $s$-colouring of $\Delta_{\chi}$. We can see from the characterization of the facets of $\Delta_{\chi}$ that each facet contains exactly one vertex from each of the sets $V_{1}^{\prime}, \ldots, V_{s}^{\prime}$, and hence $\Delta_{\chi}$ is pure of dimension $s-1$ as well as balanced.

Example 6. Let $\Delta=\left\langle x_{1} x_{2} x_{3}, x_{2} x_{4}, x_{3} x_{4}\right\rangle$ and let $\chi$ be the colouring given by $\left\{x_{1}, x_{4}\right\} \cup$ $\left\{x_{2}\right\} \cup\left\{x_{3}\right\}$. The faces of $\Delta$ are $\left\{\emptyset, x_{1}, x_{2}, x_{3}, x_{4}, x_{1} x_{2}, x_{2} x_{3}, x_{1} x_{3}, x_{2} x_{4}, x_{3} x_{4}, x_{1} x_{2} x_{3}\right\}$. These are in one-to-one correspondence with the facets of $\Delta_{\chi}$ :

$\Delta_{\chi}=\left\langle y_{1} y_{2} y_{3}, x_{1} y_{2} y_{3}, x_{2} y_{1} y_{3}, x_{3} y_{1} y_{2}, x_{4} y_{2} y_{3}, x_{1} x_{2} y_{3}, x_{2} x_{3} y_{1}, x_{1} x_{3} y_{2}, x_{2} x_{4} y_{3}, x_{3} x_{4} y_{2}, x_{1} x_{2} x_{3}\right\rangle$.

We come to the main result of this section.

Theorem 7. For any simplicial complex $\Delta$, and any s-colouring $\chi$ of $\Delta$, the simplicial complex $\Delta_{\chi}$ is vertex decomposable.

Proof. We proceed by induction on the number of vertices of $\Delta$. If $\Delta$ is the simplicial complex consisting of a single vertex $x_{1}$, then the only possible colourings of the vertices of $\Delta$ are of the form $V=V_{1} \cup \cdots \cup V_{s}$ where $V_{1}=\left\{x_{1}\right\}$ and $V_{2}, \ldots, V_{s}$ are empty. In this case $\Delta_{\chi}=\left\langle x_{1} y_{2} \ldots y_{s}, y_{1} y_{2} \ldots y_{s}\right\rangle$. This complex is vertex decomposable because $\Delta_{\chi} \backslash x_{1}=\left\langle y_{1} y_{2} \ldots y_{s}\right\rangle$ and $\operatorname{link}_{\Delta_{\chi}}\left(x_{1}\right)=\left\langle y_{2} \ldots y_{s}\right\rangle$ are both simplices.

Now suppose that $\Delta$ is a simplicial complex on the vertex set $V=\left\{x_{1}, \ldots, x_{n}\right\}$, and let $\chi$ be the $s$-colouring of $\Delta$ given by $V=V_{1} \cup \cdots \cup V_{s}$. We will show that we can decompose $\Delta_{\chi}$ by decomposing at any vertex $x_{i}$. Let $g_{1}, \ldots, g_{t}$ be the faces of $\Delta$ and define $g_{i}^{\prime}=\left\{y_{j} \mid V_{j} \cap g_{i}=\emptyset\right\}$. So $g_{1} \cup g_{1}^{\prime}, \ldots, g_{t} \cup g_{t}^{\prime}$ are the facets of $\Delta_{\chi}$.

We must show that both $\Delta_{\chi} \backslash x_{i}$ and $\operatorname{link}_{\Delta_{\chi}}\left(x_{i}\right)$ are vertex decomposable. First consider $\Delta_{\chi} \backslash x$. We assume that the facets of $\Delta_{\chi}$ are ordered so that the facets $g_{1} \cup g_{1}^{\prime}, \ldots, g_{r} \cup g_{r}^{\prime}$ do not contain the vertex $x_{i}$ and the facets $g_{r+1} \cup g_{r+1}^{\prime}, \ldots, g_{t} \cup g_{t}^{\prime}$ do contain $x_{i}$. So

$$
\Delta \backslash x_{i}=\left\{\text { faces of } \Delta \text { which do not contain } x_{i}\right\}=\left\{g_{1}, \ldots, g_{r}\right\} \text {. }
$$

Note that we are using the fact that $g_{1}, \ldots, g_{r}, g_{r+1}, \ldots, g_{t}$ is a complete list of the faces of $\Delta$ by Theorem 5 .

Without loss of generality we may assume that $x_{i} \in V_{1}$. Then $V \backslash\left\{x_{i}\right\}=\left(V_{1} \backslash\left\{x_{i}\right\}\right) \cup$ $V_{2} \cup \cdots \cup V_{s}$ is an $s$-colouring of $\Delta \backslash x_{i}$. Call this $s$-colouring $\chi^{\prime}$. Then $\left(\Delta \backslash x_{i}\right)_{\chi^{\prime}}=$ 
$\left\langle\left(g_{1} \cup g_{1}^{\prime}\right), \ldots,\left(g_{r} \cup g_{r}^{\prime}\right)\right\rangle=\Delta_{\chi} \backslash x_{i}$. Since $\Delta \backslash x_{i}$ is a simplicial complex on fewer than $n$ vertices, $\left(\Delta \backslash x_{i}\right)_{\chi^{\prime}}$ is vertex decomposable.

Now consider the link. Since $\left(g_{r+1} \cup g_{r+1}^{\prime}\right), \ldots,\left(g_{t} \cup g_{t}^{\prime}\right)$ are the facets of $\Delta_{\chi}$ which contain $x_{i}$,

$$
\begin{aligned}
\operatorname{link}_{\Delta_{\chi}}\left(x_{i}\right) & =\left\langle\left(g_{r+1} \cup g_{r+1}^{\prime}\right) \backslash\left\{x_{i}\right\}, \ldots,\left(g_{t} \cup g_{t}^{\prime}\right) \backslash\left\{x_{i}\right\}\right\rangle \\
& =\left\langle\left(\left(g_{r+1} \backslash\left\{x_{i}\right\}\right) \cup g_{r+1}^{\prime}\right), \ldots,\left(\left(g_{t} \backslash\left\{x_{i}\right\}\right) \cup g_{t}^{\prime}\right)\right\rangle .
\end{aligned}
$$

For each $1 \leqslant j \leqslant s$, set $W_{j}=\left\{x_{\ell} \in V_{j} \mid x_{\ell} \in \operatorname{link}_{\Delta}\left(x_{i}\right)\right\}$. Note that some of these sets may be empty. Then $W=W_{1} \cup \cdots \cup W_{s}$ is an $s$-colouring of $\operatorname{link}_{\Delta}\left(x_{i}\right)$. We call this $s$-colouring $\chi^{\prime \prime}$. Then

$$
\left(\operatorname{link}_{\Delta}\left(x_{i}\right)\right)_{\chi^{\prime \prime}}=\operatorname{link}_{\Delta_{\chi}}\left(x_{i}\right)
$$

and by induction $\left(\operatorname{link}_{\Delta}\left(x_{i}\right)\right)_{\chi^{\prime \prime}}$ is vertex decomposable.

The fact that $\Delta_{\chi}$ is vertex decomposable has the following consequence.

Definition 8. A pure simplicial complex $\Delta$ is shellable if there is an ordering $F_{1}, \ldots, F_{s}$ on the facets of $\Delta$ such that for all $1 \leqslant i<j \leqslant s$ there exists some $v \in F_{j} \backslash F_{i}$ and some $\ell \in\{1, \ldots, j-1\}$ with $F_{j} \backslash F_{\ell}=\{v\}$. Such an ordering on the facets is called a shelling order.

Corollary 9. For any simplicial complex $\Delta$, and any s-colouring $\chi$ of $\Delta$, the complex $\Delta_{\chi}$ is shellable (and Cohen-Macaulay). Also, any order of the facets of $\Delta_{\chi}$ which refines the order given by ordering the faces of $\Delta$ by increasing dimension is a shelling order.

Proof. By Theorem 7, $\Delta_{\chi}$ is vertex decomposable, so by [18, Corollary 2.9] it is also shellable, and consequently, Cohen-Macaulay (e.g, see [15, Theorem 8.2.6]).

For the rest, let $F_{1}, \ldots F_{s}$ be the facets of $\Delta_{\chi}$. By Theorem 5 , each $F_{i}=g_{i} \cup g_{i}^{\prime}$ where $g_{i}$ is a face of $\Delta$ and $g_{i}^{\prime}=\left\{y_{j} \mid V_{j} \cap g_{i}=\emptyset\right\}$. We order the facets $F_{1}, \ldots F_{s}$ so that $\operatorname{dim} g_{i} \leqslant \operatorname{dim} g_{j}$ if $i<j$. We now show that this is a shelling order.

Let $F_{i}, F_{j}$ be any two distinct facets of $\Delta_{\chi}$ with $i<j$. Since $i<j$, we have $\operatorname{dim} g_{i} \leqslant$ $\operatorname{dim} g_{j}$ and so there is some $x_{u} \in F_{j} \backslash F_{i}$. Since $g_{j} \backslash\left\{x_{u}\right\}$ is a face of $\Delta$ we have $g_{j} \backslash\left\{x_{u}\right\}=g_{\ell}$ for some $\ell$, and $\operatorname{since} \operatorname{dim} g_{\ell}<\operatorname{dim} g_{j}$ we have $\ell<j$. Since $g_{\ell}=g_{j} \backslash\left\{x_{u}\right\}$ we must have $F_{\ell}=g_{\ell} \cup g_{\ell}^{\prime} \in \Delta_{\chi}$ where $g_{\ell}^{\prime}=g_{j}^{\prime} \cup\left\{y_{w}\right\}$ where $x_{u} \in V_{w}$. Then

$$
F_{j} \backslash F_{\ell}=\left(g_{j} \cup g_{j}^{\prime}\right) \backslash\left(g_{\ell} \cup g_{\ell}^{\prime}\right)=\left(g_{j} \cup g_{j}^{\prime}\right) \backslash\left(\left(g_{j} \backslash\left\{x_{u}\right\}\right) \cup\left(g_{j}^{\prime} \cup\left\{y_{w}\right\}\right)\right)=\left\{x_{u}\right\} .
$$

Thus our ordering is a shelling order.

\section{Consequences: $h$-vectors and Betti numbers}

In this section, we explore some consequences of Theorem 7 for $h$-vectors. The $h$-vector $\left(h_{0}, h_{1}, \ldots, h_{d+1}\right)$ of a $d$-dimensional simplicial complex $\Delta$, denoted $h(\Delta)$, is defined in terms of the $f$-vector $f(\Delta)=\left(f_{-1}, f_{0}, \ldots, f_{d}\right)$ as follows

$$
h_{k}=\sum_{i=0}^{k}(-1)^{k-i}\left(\begin{array}{l}
d-i \\
k-i
\end{array}\right) f_{i-1}(\Delta) \text {. }
$$


We use Theorem 7 to obtain a new proof of one implication result of [3]. Note that for brevity, we have omitted the definition of a colour-shifted simplicial complex. See [12] for the definition and the discussion after the proof for some additional comments.

Theorem 10. Let $m=\left(m_{1}, \ldots, m_{t}\right) \in \mathbb{Z}_{+}^{t}$. Then the following are equivalent:

(i) $m$ is the $f$-vector of a simplicial complex.

(ii) $m$ is the $f$-vector of a colour-shifted simplicial complex.

(iii) $m$ is the $h$-vector of a balanced, vertex decomposable simplicial complex.

(iv) $m$ is the h-vector of a balanced, shellable simplicial complex.

$(v) m$ is the h-vector of a balanced, Cohen-Macaulay simplicial complex.

Proof. The equivalence of $(i),(i i),(i v)$, and $(v)$ appear in [3]. A alternative proof can be found in [1]. The implication of $(i i i) \Rightarrow(i v)$ follows from the fact that vertex decomposability implies shellabilty. Our contribution is $(i) \Rightarrow($ iii $)$. Let $f(\Delta)$ be the $f$-vector of a simplicial complex $\Delta$. For any $s$-colouring $\chi$ of $\Delta, \Delta_{\chi}$ is a balanced vertex decomposable simplicial complex by Theorems 5 and 7 . Then we have $h\left(\Delta_{\chi}\right)=f(\Delta)$. Indeed, one uses the standard technique of using the shelling of $\Delta_{\chi}$ (as given by Corollary 9) to find $h\left(\Delta_{\chi}\right.$ ) using [20, Proposition III.2.3]. One then uses the correspondence of Theorem 5 between the faces of $\Delta$ and the facets of $\Delta_{\chi}$ to relate the $h$-vector back to $f(\Delta)$. In essence, the proof of [7, Theorem 3.8] for clique-whiskering generalizes in the natural way.

Discussion 11. Theorem 10 is stated as a theorem about simplicial complexes, but [3] addressed the more general case of multi-complexes. Furthermore, in [3], colourshifted complexes are called compressed complexes. The strategy behind the orginal proof of Theorem 10 is to show that the $f$-vector of a simplicial complex is also the $f$-vector of some colour-shifted simplicial complex (the procedure for building this new colour-shifted complex is iterative in nature). Then, from this colour-shifted simplicial complex, a construction like Construction 3 is used to build a simplicial complex that is balanced and shellable whose $h$-vector is the same as the $f$-vector of the colour-shifted complex. One can show that if one applies Construction 3 to a colour-shifted simplicial complex, one has a vertex decomposable simplicial complex. Therefore, the implication (ii) $\Rightarrow$ (iii) is implicit in Theorem 10. [3, Comment 6.5] states, without proof, that one can prove $(i) \Rightarrow(i v)$ for simplicial complexes without having to pass through colourshifted complexes. One assumes that the omitted proof uses something like Construction 3 . What we have made explicit is that one can prove $(i) \Rightarrow($ iii $)$ directly without the need of colour-shifted complexes, and at the same time, identify the $f$-vectors of a simplicial complexes with a smaller subset of $h$-vectors of balanced complexes.

It has been asked whether the above statements of Theorem 10 still hold if we restrict to the class of flag complexes. In particular, Cook and Nagel [7], and Constantinescu and Varbaro [5] have posited the following conjecture (the conjecture of Cook and Nagel does not include the word balanced): 
Conjecture 12. The following equality of sets holds:

$$
\left\{\begin{array}{c}
f \text {-vectors of } \\
\text { flag complexes }
\end{array}\right\}=\left\{\begin{array}{c}
h \text {-vectors of balanced } \\
\text { vertex decomposable flag complexes }
\end{array}\right\} \text {. }
$$

The set of $f$-vectors of flag complexes has been shown to be a subset of the set of $h$-vectors of balanced vertex decomposable flag complexes. We omit the proof here, but instead point the reader to the proofs of [7, Corollary 3.10] and [5, Proposition 4.1]. The second proof is interesting since the authors use basically the same construction as Construction 3, but in the special case that the colouring is given by the partition $V=\left\{x_{1}\right\} \cup \cdots \cup\left\{x_{n}\right\}$. In some special cases, e.g., bipartite graphs (see [7]), the conjecture has been proved. We add additional evidence for Conjecture 12 in the next section.

We conclude this section by showing how to use Theorem 10 to find the graded Betti numbers of the Alexander dual of the Stanley-Reisner ideal associated to $I_{\Delta_{\chi}}$. Recall that the Stanley-Reisner ideal of $\Delta$ is the monomial ideal

$$
I_{\Delta}=\left\langle x_{i_{1}} x_{i_{2}} \cdots x_{i_{s}} \mid\left\{x_{i_{1}}, x_{i_{2}}, \ldots, x_{i_{s}}\right\} \notin \Delta\right\rangle \subseteq S=k\left[x_{1}, \ldots, x_{n}\right] .
$$

The Alexander dual of a $\Delta$, denoted $\Delta^{\vee}$, is the simplicial complex $\Delta^{\vee}=\{\bar{\sigma} \mid \sigma \notin \Delta\}$. Here, given $\sigma \subseteq\left\{x_{1}, \ldots, x_{n}\right\}$, we let $\bar{\sigma}=\left\{x_{1}, \ldots, x_{n}\right\} \backslash \sigma$. We then have:

Theorem 13. Let $\left(f_{-1}, f_{0}, \ldots, f_{d}\right)$ be the $f$-vector of a d-dimensional simplicial complex $\Delta$ on $V=\left\{x_{1}, \ldots, x_{n}\right\}$, and let $\chi$ be any s-colouring of $\Delta$. The graded Betti numbers of $I_{\Delta_{\chi}}$ in $R$ are given by the formula

$$
\beta_{i, i+n}\left(I_{\Delta_{\chi}}\right)=\sum_{j=i}^{d+1}\left(\begin{array}{l}
j \\
i
\end{array}\right) f_{j-1}(\Delta)
$$

In particular, $\operatorname{proj}-\operatorname{dim}\left(I_{\Delta_{\chi}^{\vee}}\right)=\operatorname{reg}\left(R / I_{\Delta_{\chi}}\right)=d+1$.

Proof. The projective dimension follows directly from our formula, and for the regularity, we use the identity (e.g., see [15, Proposition 8.1.10]) that $\operatorname{proj}-\operatorname{dim}\left(I_{\Delta \vee}\right)=\operatorname{reg}\left(R / I_{\Delta}\right)$.

Because $\Delta_{\chi}$ is pure and vertex decomposable (and thus shellable), [9, Corollary 5$]$ gives

$$
\sum_{i \geqslant 1} \beta_{i}\left(R / I_{\Delta_{\chi}^{\vee}}\right) t^{i-1}=\sum_{i \geqslant 0} h_{i}\left(\Delta_{\chi}\right)(t+1)^{i}
$$

Note that in [9], the authors are taking the resolution of $R / I_{\Delta_{\chi}^{\vee}}$, so $\beta_{i}\left(R / I_{\Delta_{\chi}^{\vee}}\right)=\beta_{i-1}\left(I_{\Delta_{\chi}^{\vee}}\right)$. Furthermore, although the formula of [9] is expressed in terms of total graded Betti numbers, the resolution of $I_{\Delta_{\chi}^{\vee}}$ is linear (this is because $\Delta_{\chi}$ is shellable and pure of dimension $s-1$, and hence $I_{\Delta_{\chi}}$ is generated in degree $n$ and is componentwise linear, which implies the ideal has a linear resolution). We therefore have $\beta_{i-1}\left(I_{\Delta_{\chi}^{\vee}}\right)=\beta_{i-1, n+i-1}\left(I_{\Delta_{\chi}^{\vee}}\right)$.

To finish the proof, Theorem 10 allows us to replace $h_{i}\left(\Delta_{\chi}\right)$ with $f_{i-1}(\Delta)$ in the formula (1), thus giving the desired formula for $\beta_{i-1, n+i-1}\left(I_{\Delta_{\chi}^{\vee}}\right)$. 
Remark 14. For any valid $f$-vector $f(\Delta)=\left(f_{0}, \ldots, f_{d}\right)$, the sequence

$$
\left(\sum_{j=0}^{d+1}\left(\begin{array}{l}
j \\
0
\end{array}\right) f_{j-1}(\Delta), \sum_{j=1}^{d+1}\left(\begin{array}{l}
j \\
1
\end{array}\right) f_{j-1}(\Delta), \ldots, \sum_{j=d+1}^{d+1}\left(\begin{array}{c}
j \\
d+1
\end{array}\right) f_{j-1}(\Delta)\right)
$$

is a valid sequence of Betti numbers for an ideal with a linear resolution by Theorem 13 . Herzog, Sharifan, and Varbaro [17] classified all valid sequences of Betti numbers for an ideal with a linear resolution. While one can deduce that (2) is valid sequence of Betti numbers from [17], our Theorem 13 highlights how to start with a simplicial complex with a given $f$-vector, and find a square-free monomial ideal whose graded linear resolution has Betti sequence given by (2). This contrasts with [17] since the ideal they construct with Betti sequence (2) need not be a square-free monomial ideal.

\section{Application: independence complexes of chordal graphs}

In this section we provide new evidence for Conjecture 12. We give a condition to "reverse" Construction 3, and then apply it to independence complexes of chordal graphs.

The restriction of $\Delta$ to $W \subseteq V$ is the subcomplex $\left.\Delta\right|_{W}=\{F \in \Delta \mid F \subseteq W\}$. Our criterion for "reversing" the process of the last section requires the following terminology.

Definition 15. Suppose $\Delta=\left\langle F_{1}, \ldots, F_{s}\right\rangle$ is a simplicial complex on the vertex set $V$. We say that $\Delta$ has a facet restriction with respect to $F$ if $F$ is a facet of $\Delta$ such that

$$
\left.\Delta\right|_{V \backslash F}=\left\{F_{1} \backslash F, \ldots, F_{s} \backslash F\right\} .
$$

Note that the inclusion $\left.\Delta\right|_{V \backslash F} \supseteq\left\{F_{1} \backslash F, \ldots, F_{s} \backslash F\right\}$ always holds; however, in general the two sets may not be equal as we see in the following example.

Example 16. Let $\Delta=\langle 123,234,345,456\rangle$ (to simplify notation, we are writing $i$ for $x_{i}$ ). By considering each facet of $\Delta$, we can show it has no facet restriction. Let $F$ be the facet 123 . Then

$\left.\Delta\right|_{V \backslash F}=\{\emptyset, 4,5,6,45,56,46,456\} \neq\{123 \backslash F, 234 \backslash F, 345 \backslash F, 456 \backslash F\}=\{\emptyset, 4,45,456\}$.

Similarly, if we consider the facet 234 we see that

$\left.\Delta\right|_{V \backslash 234}=\left.\Delta\right|_{156}=\{\emptyset, 1,5,6,56\} \neq\{123 \backslash 234,234 \backslash 234,345 \backslash 234,456 \backslash 234\}=\{1, \emptyset, 5,56\}$.

By symmetry, the facets 345 and 456 also fail to give a facet restriction. Therefore the simplicial complex $\Delta$ has no facet restriction.

Example 17. Let $\Delta$ be the simplicial complex $\langle 124,245,235,456\rangle$. Then $\Delta$ has a facet restriction with respect to the facet 245 since

$$
\left.\Delta\right|_{V \backslash 245}=\left.\Delta\right|_{136}=\{\emptyset, 1,3,6\}=\{124 \backslash 245,245 \backslash 245,235 \backslash 245,456 \backslash 245\} .
$$


The existence of a facet restriction allows us to "reverse" our proof of Theorem 10 (i) $\Rightarrow($ iii $)$. In particular, we show that if $\Delta$ has facet restriction, we can make a new simplicial complex whose $f$-vector is the same of $h(\Delta)$.

Theorem 18. Let $\Delta=\left\langle F_{1}, \ldots, F_{t}\right\rangle$ be a pure, balanced simplicial complex such that $\Delta$ has a facet restriction with respect to the facet $F$. Then $\Delta=\left(\left.\Delta\right|_{V \backslash F}\right)_{\chi}$ where $\chi$ is the colouring induced from the colouring of $\Delta$. In particular, $\Delta$ is vertex decomposable and $h(\Delta)=f\left(\left.\Delta\right|_{V \backslash F}\right)$.

Proof. Let $d-1$ be the dimension of $\Delta$. Because $\Delta$ is pure and balanced, the colouring $\chi$ is given by a partition $V=V_{1} \cup V_{2} \cup \cdots \cup V_{d}$ such that $\left|F_{j} \cap V_{i}\right|=1$ for all $1 \leqslant j \leqslant t$ and $1 \leqslant i \leqslant d$. After relabelling, we can assume that $F_{1}$ is the facet that gives the facet restriction. Note that $\left.\Delta\right|_{V \backslash F_{1}}$ is a simplicial complex on $Y=V \backslash F_{1}$, and is $d$-colourable since $\left.\Delta\right|_{V \backslash F_{1}}$ inherits a colouring from $\chi$ given by:

$$
Y=V \backslash F_{1}=\left(V_{1} \backslash F_{1}\right) \cup\left(V_{2} \backslash F_{1}\right) \cup \cdots \cup\left(V_{d} \backslash F_{1}\right) .
$$

Abusing notation, let $\chi$ denote this new colouring. Then $\left(\left.\Delta\right|_{V \backslash F_{1}}\right)_{\chi}$ is a balanced vertex decomposable simplicial complex such that $h\left(\left(\left.\Delta\right|_{V \backslash F_{1}}\right)_{\chi}\right)=f\left(\left.\Delta\right|_{V \backslash F_{1}}\right)$ by Theorem 10 .

To complete the proof, it suffices to show that $\left(\left.\Delta\right|_{V \backslash F_{1}}\right)_{\chi}$ and $\Delta$ are the same simplicial complexes, but with a different labelling of the vertices. By Theorem 5, the facets of $\left(\left.\Delta\right|_{V \backslash F_{1}}\right)_{\chi}$ are in one-to-one correspondence with the faces of $\left.\Delta\right|_{V \backslash F_{1}}$. But we also have that the facets of $\Delta$ are in one-to-one correspondence with the faces of $\left.\Delta\right|_{V \backslash F_{1}}$ via the map $F_{i} \mapsto F_{i} \backslash F_{1}$. Indeed, this map is clearly onto by our assumption that $\Delta$ has a facet restriction with respect to $F_{1}$. It suffices to show that this map is one-to-one. So, suppose $F_{i} \backslash F_{1}=F_{j} \backslash F_{1}$, but $F_{i} \neq F_{j}$. This means that there is a vertex $x \in F_{i} \backslash F_{j}$ because the simplicial complex is pure. Since $\Delta$ is balanced, there is a vertex $y \in F_{j} \backslash F_{i}$ with the same colour as $x$. Because $F_{i} \backslash F_{1}=F_{j} \backslash F_{1}$, we must have $x$ and $y$ in $F_{1}$. But this contradicts the colouring of $\Delta$. By combining these two one-to-one correspondences, we get the desired bijection between the facets of $\Delta$ and $\left(\left.\Delta\right|_{V \backslash F_{1}}\right)_{\chi}$.

Example 19. In Example 17 we saw that $\Delta=\langle 124,245,235,456\rangle$ has a facet restriction with respect to the facet 245 . Since $\left.\Delta\right|_{136}=\{\emptyset, 1,3,6\}$, the $f$-vector of $\left.\Delta\right|_{136}$ is $f\left(\left.\Delta\right|_{136}\right)=$ $(1,3)$. Therefore the $h$-vector of $\Delta$ is $h(\Delta)=f\left(\left.\Delta\right|_{136}\right)=(1,3)$.

Recall that a graph $G$ is chordal if every induced cycle of $G$ of length $\geqslant 4$ has a chord. We will prove the following fact about the independence complexes of chordal graphs.

Lemma 20. Let $\Delta=\operatorname{Ind}(G)$ be the independence complex of a chordal graph $G$. If $\Delta$ is also pure, then $\Delta$ has a facet restriction.

Theorem 18 and Lemma 20 combine to prove a special case of Conjecture 12.

Theorem 21. We have the the following equivalence of sets:

$$
\left\{\begin{array}{c}
f \text {-vectors of independence } \\
\text { complexes of chordal graphs }
\end{array}\right\}=\left\{\begin{array}{c}
h \text {-vectors of balanced, vertex decomposable } \\
\text { independence complexes of chordal graphs }
\end{array}\right\} \text {. }
$$


Proof. If $f(\Delta)$ is the $f$-vector of $\Delta=\operatorname{Ind}(G)$ when $G$ is chordal, then for any colouring $\chi$ of $\Delta=\operatorname{Ind}(G)$, the simplicial complex $\Delta_{\chi}$ is balanced and vertex decomposable by Theorem 7 , and $f(\Delta)=h\left(\Delta_{\chi}\right)$ by Theorem 10 . It remains to explain why $\Delta_{\chi}$ is the independence complex of a chordal graph. As noted in Remark 4, in the case of independence complexes, Construction 3 is the same as the clique wiskering construction of Cook and Nagel [7]. Furthermore, the clique wiskering construction of a chordal graph produces a new chordal graph, so $\Delta_{\chi}$ is the independence complex of a chordal graph.

To show the reverse containment, let $G$ be any chordal graph such that $\Delta=\operatorname{Ind}(G)$ is balanced and vertex decomposable. Because $\Delta$ is vertex decomposable, and thus pure, by Lemma $20, \Delta$ has a facet restriction with respect to some facet $F$. Then by Theorem 18 , we have $h(\Delta)=f\left(\left.\Delta\right|_{V \backslash F}\right)$. To complete the argument, we note that

$$
\left.\Delta\right|_{V \backslash F}=\left.\operatorname{Ind}(G)\right|_{V \backslash F}=\operatorname{Ind}\left(\left.G\right|_{V \backslash F}\right) .
$$

The graph $\left.G\right|_{V \backslash F}$ is an induced subgraph of a chordal graph, and so is a chordal graph. So $h(\Delta)=f\left(\operatorname{Ind}\left(\left.G\right|_{V \backslash F}\right)\right)$, thus completing the proof.

To prove Lemma 20 we will require a result of Herzog, Hibi, and Zheng about the clique complex. For any finite simple graph $G=\left(V_{G}, E_{G}\right)$ the clique complex of $G$ is the simplicial complex $C l(G)=\left\{C \subseteq V|G|_{C}\right.$ is a clique $\}$. Below, a vertex $v \in V$ is a free vertex if $v$ is contained in exactly one facet of $\Delta$.

Theorem 22 ([16, Theorem 2.1]). Let $G$ be a chordal graph and let $C_{1}, \ldots, C_{t}$ be all the facets of $\mathrm{Cl}(G)$ that contain a free vertex. The following are equivalent:

(a) $G$ is unmixed, i.e., all maximal independent sets have the same cardinality.

(b) $V=C_{1} \cup C_{2} \cup \cdots \cup C_{t}$ is a partition of the vertices of $G$.

We are now ready to prove Lemma 20.

Proof. (of Lemma 20) Let $\Delta=\operatorname{Ind}(G)$ be the independence complex of a chordal graph, and furthermore, assume $\Delta$ is pure. Let $C_{1}, \ldots, C_{t}$ be the facets of $C l(G)$ which contain a free vertex. Since $\Delta$ is pure, we know that $G$ is unmixed. Thus by Theorem 22, we have the partition $V=C_{1} \cup \cdots \cup C_{t}$. For $1 \leqslant i \leqslant t$, let $y_{i}$ be a free vertex of $C l(G)$ contained in $C_{i}$. Set $F=\left\{y_{1}, \ldots, y_{t}\right\}$. We will show that $F$ is a facet of $\Delta$ and that $\Delta$ has a facet restriction with respect to $F$.

It is clear that $F=\left\{y_{1}, \ldots, y_{t}\right\}$ is an independent set since each $y_{i}$ is in a unique maximal clique $C_{i}$ and an edge $\left\{y_{i}, y_{j}\right\}$ would constitute a clique of size 2 . Further, $F$ is a maximal independent set since every vertex $x \notin F$ is in some $C_{i}$ and therefore adjacent to some $y_{i}$. Since $\Delta$ is assumed to be pure, this means that every facet has size $t$.

Next let $F_{1}, \ldots, F_{s}$ be the facets of $\Delta$. To finish the proof we will show that

$$
\left.\Delta\right|_{V \backslash F}=\left\{F_{1} \backslash F, \ldots, F_{s} \backslash F\right\} .
$$

We simply need to show $\left.\Delta\right|_{V \backslash F} \subseteq\left\{F_{1} \backslash F, \ldots, F_{s} \backslash F\right\}$. Let $\left.H \in \Delta\right|_{V \backslash F}$, and define $H^{\prime}=H \cup\left\{y_{i} \mid C_{i} \cap H=\emptyset\right\}$. Then $H^{\prime}$ is independent since the neighbours of $y_{i}$ are the elements of $C_{i} \backslash\left\{y_{i}\right\}$. Since $H^{\prime}$ has cardinality $t$, it is a facet of $\Delta$. Therefore $H=H^{\prime} \backslash F$ which proves that $\left.\Delta\right|_{V \backslash F}=\left\{F_{1} \backslash F, \ldots, F_{s} \backslash F\right\}$. 


\section{References}

[1] E. Babson and I. Novik. Face numbers and nongeneric initial ideals. Electron. J. Combin. 11(2), 2004-6.

[2] J. Biermann, C. Francisco, T. Hà, and A. Van Tuyl. Colorings of simplicial complexes and vertex decomposability. Preprint, 2012. arXiv:1209.3008v1.

[3] A. Björner, P. Frankl, and R. Stanley. The number of faces of balanced CohenMacaulay complexes and a generalized Macaulay theorem. Combinatorica, 7(1): 23-34, 1987.

[4] CoCoATeam, CoCoA: a system for doing Computations in Commutative Algebra. Available at http://cocoa.dima.unige.it.

[5] A. Constantinescu and M. Varbaro. On the $h$-vectors of Cohen-Macaulay flag complexes. To appear in Math. Scand., 2010. arXiv:1004.0170v2.

[6] D. Cook II. Simplicial Decomposability. JSAG, 2: 20-23, 2010.

[7] D. Cook II and U. Nagel. Cohen-Macaulay graphs and face vectors of flag complexes. SIAM J. Discrete Math., 26(1): 89-101, 2012.

[8] A. Dochtermann and A. Engström. Algebraic properties of edge ideals via combinatorial topology. Electron. J. Combin. , 16(2), 2009.

[9] J. Eagon and V. Reiner. Resolutions of Stanley-Reisner rings and Alexander duality. J. Pure Appl. Algebra, 130(3): 265-275, 1998.

[10] C. Francisco and T. Hà. Whiskers and sequentially Cohen-Macaulay graphs. J. Combin. Theory Ser. A, 115(2): 304-316, 2008.

[11] A. Frohmader. How to construct a flag complex with a given face vector. Preprint, 2011. arXiv:1112.6061v1.

[12] A. Frohmader, Flag f-vectors of colored complexes. J. Combin. Theory Ser. A 119 (2012), no. 5, 937-941.

[13] D. Grayson and M. Stillman. Macaulay 2, a software system for research in algebraic geometry. http://www. math.uiuc.edu/Macaulay2/.

[14] G. Hetyei. Central Delannoy numbers and balanced Cohen-Macaulay complexes. Ann. Comb., 10(4): 443-462, 2006.

[15] J. Herzog and T. Hibi. Monomial ideals. Graduate Texts in Mathematics, no. 260. Springer-Verlag, 2011.

[16] J. Herzog, T. Hibi, and X. Zheng. Cohen-Macaulay chordal graphs. J. Combin. Theory Ser. A, 113(5): 911-916, 2006.

[17] J. Herzog, L. Sharifan, and M. Varbaro. Graded Betti numbers of componentwise linear ideals. To appear Proc. Amer. Math. Soc., 2011. arXiv:1111.0442v1.

[18] J. Provan and L. Billera. Decompositions of simplicial complexes related to the diameters of convex polyhedra. Math. Oper. Res. 5(4):576-594, 1980. 
[19] R. Stanley. Balanced Cohen-Macaulay complexes. Trans. Amer. Math. Soc. 249(1): 139-157, 1979.

[20] R. Stanley. Combinatorics and Commutative Algebra, Second Edition. Birkhäuser, Boston, 1983.

[21] R. H. Villarreal. Cohen-Macaulay graphs. Manuscripta Math. 66(3): 277-293, 1990.

[22] R. Woodroofe. Vertex decomposable graphs and obstructions to shellability. Proc. Amer. Math. Soc. 137(10): 3235-3246, 2009. 